\title{
Vinculación con la sociedad aplicada, paradigma en la educación superior
}

\section{Entailment with society, a paradigm in higher education}

\section{RESUMEN:}

El documento a continuación, describe como la vinculación con la sociedad aplicada puede convertirse en una herramienta de transformación social muy poderosa, cuando es utilizada de manera acertada. Establece el verdadero significado de un paradigma dentro de la educación superior, para ser replicado más no copiado. La Universidad UTE detectó en su momento la oportunidad de intervenir en un territorio que se vio afectado drásticamente por el sismo en Ecuador, que tuvo lugar en abril del 2016, aquí explotó la situación de manera positiva con el fin de generar un proyecto integral de vinculación con la sociedad que permita la generación de propuestas urbano-arquitectónicas integrales, como modelos de reconstrucción para el territorio afectado. Las metodologías que se utilizaron para desarrollar este proyecto fueron variadas, sin embargo entre las más destacadas tenemos: Cuantitativa, aquí se amplió los datos obtenidos de varias fuentes locales y estatales. Cualitativa, con enfoques subjetivos e individuales a través de la interpretación, observación, relatos y entrevistas propias de la población; y la sintética, con el objetivo de profundizar en los análisis de una forma metódica para alcanzar una compresión total de cada grupo integrante y sus particularidades, con esto se buscó comprender la conducta total integral de la población. Fundamentado en estas metodologías, se logró determinar los componentes y lineamientos que se convertirían en las directrices del proyecto final. El artículo posee conclusiones que se han podido obtener a la fecha actual, demostrando la importancia de contar con principios transformadores de la sociedad desde la academia, a través de individuos que poseen criterios propositivos, respaldando una formación profesional integral sustentada en conocimientos tangibles y plasmados, apuntalados siempre por una investigación científica con una profunda reflexión crítica sobre su práctica.

Palabras clave: herramienta, integral, sociedad, tangible, transformación, vinculación. 


\section{ABSTRACT:}

The document below describes how applied entailment with society can become a very powerful tool for social transformation, when it is used correctly. It establishes the true meaning of a paradigm within higher education, to be replicated rather than copied. University UTE detected an opportunity to intervene in a territory that was drastically affected by the earthquake in Ecuador that took place in April 2016, here exploited the situation in a positive way in order to generate a comprehensive project of entailment with society that allows the generation of integral urban-architectural proposals as models of reconstruction for the affected territory. The methodologies that were used to develop this project were varied, however among the most important we have the quantitative, here we expanded data obtained from various local and state sources. The qualitative with subjective and individual approaches through the interpretation, observation, stories and interviews of the population; and the synthetic with the aim of deepening the analysis in a methodical way to achieve a total understanding of each group and its particular characteristics, this was sought to understand the overall behavior of the population. Based on these methodologies, it was possible to determine the components and guidelines that would become the basis of the final project. The article concludes with recommendations that have been obtained to the current date, demonstrating the importance of having transforming principles of society from academia, through individuals who possess proactive criteria, supporting a comprehensive professional training based on tangible knowledge and embodied, always underpinned by a scientific investigation with a deep critical reflection on its practice.

Key words: tool, integral, society, tangible, transformation, entailment.

\section{INTRODUCCIÓN}

En la actualidad el uso del término paradigma ha sido sobre explotado, llegando de cierta manera, inclusive, a erosionarlo. Esta aseveración se la puede verificar en distintos ámbitos, desde el entorno discursivo de la investigación, hasta ciertas disciplinas que abordan temas sociales. A lo largo del tiempo gran parte de espacios académicos y, en particular, entidades que se encuentran a cargo de formar profesionales, han demostrado que dentro de sus mallas o planes de estudio incluyen materias relacionadas a la "Investigación", o temáticas que se acercan a aquello. Dentro de sus contenidos se puede apreciar el uso reiterado y muchas veces inapropiado sobre el término paradigma. Es por esto, que se debe redirigir y tomar con mayor precaución este enfoque, con el único objetivo de recuperar su verdadero sentido y significado. (Murray, 1992)

Para generar una comprensión más profunda sobre paradigma y su aplicabilidad, en este caso, relacionado a un experimento académico, es importante revisar la etimología y aparición de este término. Paradigma deriva su origen del griego

paradigma, esto significa: ejemplo, modelo o patrón; originalmente, Platón aplicó esta definición con el objetivo de plasmar o transmitir la idea de mediación, que existe entre la realidad y su concepción o interpretación. Justamente en su obra plantea la idea de un mundo inteligible, como la auténtica realidad, definiendo esto, con un cierto tinte o carácter religioso que deriva consecuencias en diferentes campos tales como la epistemología, ética y política. Por otro lado Platón también aborda el mundo sensible, en este entorno la concepción es opuesta, todos los elementos tienen un carácter temporal, cambiante,

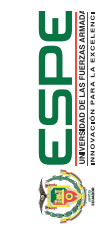


corruptible y espacial. El cuerpo de los seres humanos es incluido en este mundo, aquí no hay cabida para la ciencia simplemente existe espacio para la opinión. (Grunaguer, 2010)

Con este fundamento el reto platónico es la base para el inicio del experimento que se plantea sobre la vinculación relacionada a la reconstrucción o recuperación en territorios que han sufrido catástrofes. Las propuestas que se generen a raíz de este experimento serán plasmadas a través de proyectos integrales multifacéticos. Estos, deberán exhibir de manera constante el vínculo entre ese mundo inteligible, conceptual, ideático y el material o sensible. Sustentados, una vez más, en la teoría de Platón, surgen tres pilares que apoyarán y llevarán adelante este experimento:

- Participación, la idea como propiedad intrínseca de la cosa.

- Presencia, como cualidad circunstancial de la cosa.

- Imitación, entre las Ideas y las cosas en que resplandecen sus cualidades. Se da una relación análoga a la existente entre el modelo y su imagen.

Es por esto que de acuerdo a las teorías y el material analizado se puede definir que el verdadero significado de un paradigma va más allá que un modelo común, que se lo interpreta como una "copia", "patrón" o "muestra" de algo existente. Un paradigma debe ser considerado como modelo ejemplar, casi perfecto, con el objetivo que se lo pueda considerar digno de ser seguido, mejorado, más no copiado. En el artículo a continuación se presentará una experiencia puntual real sobre un proyecto de vinculación aplicado en la Universidad UTE, sU concepción, resultados y análisis.

\section{MARCO TEÓRICO}

Con el fundamento y panorama claro sobre la definición tangible de paradigma que guía y direcciona la visión, es importante abordar la vinculación con la sociedad. La vinculación comprendida desde un término jurídico e historiográfico, define básicamente la posición de propiedad o pertenencia de algo.

En el Antiguo Régimen significaba: "sujeción de los bienes para perpetuarlos en un determinado empleo y en el orden sucesorio dispuesto por el fundador del vínculo....se denominan "vinculaciones" el conjunto de instituciones jurídicas que convierten en inalienables los bienes a los que afectan." (Tellería, 2012)

Sin embargo, es importante aclarar que esta definición original sobre vinculación sufre una transformación o evolución, por así llamarlo de alguna manera, con la revolución industrial, etapa en la historia de la humanidad que jugó un papel fundamental, "la industria y las actividades manufactureras sustituyeron al trabajo manual. Esto supuso la mecanización de múltiples procesos productivos y la eliminación de numerosos puestos de trabajo, ya que dichas tareas pasaron a ser realizadas por máquinas." Esta ruptura entre la mano de obra y la producción, como resultado de la revolución, provoca una disolución de los vínculos que afectaban únicamente a los bienes materiales, dando paso a un escenario apropiado para la aparición de una serie de transformaciones socioeconómicas y culturales que permiten a la sociedad establecer nexos intangibles, simbólicos, espirituales, sociales, vivenciales. La vinculación, en la mayoría de ocasiones, no tiene nada que ver con la proximidad o el contacto físico. (Montoya, 2014) 
En la sociedad actual, analizar a profundidad aspectos relacionados a la universidad y sus lazos hacia su entorno inmediato es considerado un reto. Esto debido a que, adicional al sentimiento de voluntad que se requiere, demanda también un profundo conocimiento de la historia de los pueblos en los cuales se va a trabajar. "La universidad está comprometida con la sociedad, es agente de cambio capaz de generar los conocimientos teóricos y prácticos que elevan la creación y recreación de sus individuos, necesarios para la solución de problemas a través de proyectos que estimulen la interacción recíproca universidad-comunidad, desarrollando la vida nacional en todos sus aspectos." (Ricardo Jordán, 2011 1)

Sin embargo, se debe resaltar y comprender que lastimosamente la academia ha sido vista como fábrica de soluciones "inmediatas y milagrosas", tanto de parte de la sociedad como de los gobiernos. Si bien es cierto que los institutos de educación superior poseen distintas especialidades y enfoques, por la variedad de profesionales que forman, y por consiguiente tienen mucho potencial para brindar soluciones integrales a los distintos problemas que son parte del Estado, pero no necesariamente involucra el que posean intereses en común. En reiteradas ocasiones se ha evidenciado que los planes de los gobiernos no responden necesariamente de manera prioritaria al interés de la "sociedad". Estos distintos enfoques en las prioridades han desarrollado que cada actor genere sus propios proyectos y agendas, con una carencia absoluta de coordinación que permita trabajar en conjunto y compartiendo los mismos objetivos. (Rocchio, 2019)

Es en este sentido, a través de la Ley Orgánica de Educación Superior (LOES) se genera una figura, interna en las Universidades, denominada
Vinculación con la Sociedad. El objetivo de esta "extensión" de los institutos de educación superior es brindar un nexo para permitir un trabajo de sinergia entre el Estado y la academia. Con el tiempo y la experiencia obtenida en los distintos proyectos aplicados, se ha determinado que la Vinculación con la sociedad puede ser una herramienta político- académica muy poderosa, que crea nuevas oportunidades para llevar la teoría a la práctica, llegando a generar la apertura adicional hacia la investigación.

Compaginando esta aplicación práctica con las vivencias y experiencias de los distintos actores en las comunidades, sujetos de las intervenciones, se logra alcanzar y vivenciar a plenitud la democracia, una democracia pura, transparente, cuyo verdadero interés es el bien común, ya que todos los proyectos son pensados y diseñados por y para las mismas comunidades. Esto además garantiza la creación de sentimientos de identidad y apropiación dentro de las poblaciones beneficiadas. (Moya, 2019)

Tomando como punto de partida esta concepción de la vinculación con la sociedad, los parámetros que deberían ser los cimientos dentro de la educación superior, serían los siguientes ejes fundamentales:

- Conocimiento basado en la convivencia y cercanía con la sociedad que requiere la ayuda.

- Respeto ante los saberes ancestrales y las organizaciones comunitarias.

- Tomar en cuenta las demandas sociales como la base para el planteamiento sostenible y responsable a través de la investigación científica y la participación activa de todos los actores. 
Estos lineamientos buscan incentivar un trabajo interdisciplinario cuyos valores estén basados principalmente en objetivos participativos, incluyentes, solidarios, cooperativos, políticos, técnicos y científicos. La vinculación con la sociedad no puede, ni deber ser confundida con asistencialismo, subsidio o voluntariado. Es un trabajo a largo plazo, fundamentado en proyectos sostenibles que buscan despertar el sentimiento de trabajo colaborativo en las poblaciones, al mismo tiempo que van creando modelos socio-económicos replicables y constantemente mejorables.

El objetivo es que sea concebido como una herramienta de interacción constante entre la academia y su entorno, que sensibilice a los futuros profesionales sobre la importancia de la participación y la solidaridad. Generando, a través de esta ideología, sociedades más equitativas e igualitarias, desnudando la realidad sobre la vulnerabilidad de ciertas poblaciones en nuestro país. Países en vías de desarrollo, tal como el caso del Ecuador, en la mayoría de ocasiones no cuentan con los recursos suficientes para satisfacer los programas que el gobierno central plantea, por lo tanto buscan apoyo de entidades externas. Es aquí que las Universidades intervienen y logran aportar significativamente, transformando políticas, relaciones sociales y económicas. Estos aportes deberían ir siempre fundamentados en valores propios de cada nación, en el caso del Ecuador por ejemplo, deberán perseguir interculturalidad, democracia, autodeterminación, bien común y pertinencia.

Citando a Nelson Mandela de acuerdo a lo expuesto en su discurso político social que le ayudó a sobrellevar la persecución de la cual fue sujeto indica: "Cuando un hombre ha hecho lo que él considera como su deber para con su pueblo y su país, puede descansar en paz." Este debe ser el lema de todo instituto de educación que quiera ser parte de cualquier proceso de Vinculación con la sociedad. Se necesita actores seriamente comprometidos que no busquen un beneficio político o individual, se requiere gente que tenga como único fin alcanzar el bien común para el desarrollo de la sociedad como tal. (Porreca, 2019)

\section{METODOLOGÍA}

Para ejecutar este trabajo se ha utilizado distintas metodologías de investigación, sin embargo, las dos principales han sido sintética y cuantitativa. En el caso de la sintética se la utilizó a través del proceso de razonamiento que buscó reconstruir el todo del lugar, que fue tomado como caso de trabajo de vinculación generando un antes y después. Aquí se distinguió elementos y factores a través del análisis de la información que se levantó en sitio; esto fue ejecutado más como un procedimiento mental cuyo fin era la comprensión integral de la esencia de la población, sus partes y particularidades. (Rojo, 2011 ).

En esta etapa del proyecto se buscó reconstruir y comprender los aspectos y relaciones básicas en una perspectiva total. Adicional se aplicó también el método cuantitativo, esto sustentado en una investigación empírico-analista. Se recopiló varios datos estadísticos de fuentes públicas y privadas, que permitieron dar algunas respuestas a causas-efectos concretas. Aquí se generó respuestas de la población a preguntas específicas, que fueron elaboradas a través de encuestas y entrevistas. Con los resultados que se obtuvo, se logró el lanzamiento de todos los nuevos componentes propios del proyecto. 


\section{San José de Chamanga, el proyecto UTE de recuperación}

La Universidad UTE decide tomar cartas en el asunto y aborda la problemática existente en el Ecuador aportando de alguna manera con este proceso de cambio radical del sistema. En función con esta mentalidad la Universidad UTE concibe a la vinculación con la sociedad como el proceso sustantivo orientado a generar un sistema integrador entre la universidad y su entorno, que apoya la investigación, gestión académica y docencia, a través de la ejecución de programas y proyectos multidisciplinarios de desarrollo, formulados con una visión sistémica, que fortalezca la creación y aplicación de soluciones viables, innovadoras y sustentables que propicien el intercambio del conocimiento para generar un impacto positivo en el desarrollo sostenible del país. La Facultad de Arquitectura y Urbanismo, desde su visión técnica-profesional, decide aportar con este proceso de vinculación pero con un enfoque tangible y real. Deja de lado proyectos de pequeña escala y decide aportar con una propuesta seria de mejora para una población de la costa ecuatoriana afectada por el sismo de abril 2016. Así se determina que la parroquia de San José de Chamanga es la que cumple con el perfil ideal para el proyecto. (Moya, 2019)

La parroquia de San José de Chamanga es una población de la costa ecuatoriana que fue afectada en su totalidad, desde el punto de vista espacial, social, económico, productivo y administrativo. La situación que se generó en el post-catástrofe, fue de un gran porcentaje del parque construido dañado a diferentes niveles (completamente destruido o derruido, gravemente dañado, dañado o levemente dañado). La vulnerabilidad de lo construido en relación a las condicionantes que conlleva la cercanía con el estuario del río Cojimíes (licuefacción del suelo, etc.) fue un factor olvidado, que pasó a un segundo plano, aportando a la afectación de este asentamiento urbano. Si a esto se añade que San José de Chamanga, en su post-catástrofe recibió intervenciones antrópicas inadecuadas, lo único que se logró fue el incremento de problemas provenientes de intervenciones urbanas y edificatorias sin ningún tipo de planificación que, aunque se llevaron a cabo con la intención de aportar soluciones, generaron otro tipo de problemas, como segregación socio-espacial, agresión a los aspectos sociales, culturales y marginación de la economía autóctona. En este escenario la catástrofe se la consideró como una "oportunidad" para desarrollar un modelo estratégico capaz de planificar procesos de capacitación para la transferencia de tecnología y metodología, con el fin de reconstruir adecuadamente el entorno edificado y en específico la vivienda, con enfoques puntuales a los elementos arquitectónico de equipamiento que estructura el plan urbano. (Rocchio, 2019)

La Facultad de Arquitectura y Urbanismo diseñó una visión basada en la identidad del contexto específico. Tal visión se fundamenta, además de otros aspectos, en un análisis morfo-tipológico del hábitat identitario de las cinco áreas identificadas durante el workshop internacional de arquitectura equinoccial (WinAReQ) del mes de junio 2016 donde se evidenció la necesidad de una reconstrucción post-catástrofe sostenible y resiliente.

La visión de la reconstrucción de las viviendas se estructura de acuerdo a tres grandes ejes, la autoconstrucción, la autoconstrucción asistida y la asistencia técnica. Con estos se puede concluir que en los dos 
primeros se busca una capacitación de la población que opta por la construcción de su propia vivienda, por otro lado, el tercer bloque se enfoca en un aporte y/o desarrollo de la tecnología y cultura constructiva del lugar. Además, este planteamiento se concibe como un proceso en el que se considera la posibilidad de presentarse diferentes escenarios con distintas fases temporales, y el cual se va adaptando a posibles realidades que se generan en la parroquia. Esta visión permitirá generar un proceso que considera y se adapta a una realidad cambiante. (Porreca, 2019)

A lo largo del tiempo la Universidad UTE ha realizado varias visitas a la parroquia realizando el levantamiento de informaciones y talleres participativos que han permitido definir las necesidades específicas en relación a vivienda y equipamientos. Cabe resaltar que instituciones como la Universidad Católica de Chile y el Politécnico di Milano han evidenciado tales necesidades y han apoyado con decisión los lineamientos estratégicos generales definidos por la Facultad de Arquitectura y Urbanismo de la Universidad UTE; un trabajo de equipo muy importante que ha visto la participación de la carrera al evento de Naciones Unidas Hábitat III a través de un Side Event y un Networking Event, basados en proyectos de mutua colaboración en temas de estudios urbanos y reconstrucción post-catástrofe.

Alrededor de 4500 personas $^{2}$, vivieron en carpas formales, informales o de invasión. Muchas de estas personas no han abandonado aún estos refugios y siguen ocupándolos como sus hogares (Amaya, 2014). La clave para llegar a una exitosa planificación urbana son las estrategias de desarrollo urbano integral que se generen sobre la base de diferentes categorías que logren garantizar un correcto y equilibrado proceso de trabajo que se adapte a los cambios y a las necesidades que continuamente van evolucionando y por consiguiente modificando.

El objetivo del proyecto de Vinculación planteado por la Facultad de Arquitectura de la UTE, es facilitar un desarrollo equilibrado de Chamanga y su población. Este producto constará de diagnóstico, visión estratégica de intervención, diseño de la propuesta urbana, espacios públicos y áreas verdes, todo cerrará con una socialización y validación final de parte de todos los actores involucrados.

Cabe resaltar que este proyecto es multidisciplinario, cuenta con la participación de distintas carreras tales como Educación Inicial, Restauración y Museología, Periodismo, Diseño Gráfico Publicitario, Relaciones Publicas, Ingeniería Automotriz y Ciencias administrativas, esto con el objetivo de aprovechar diferentes áreas de conocimiento para aportar ayudas concretas mediante la creación de estrategias, redacción de programas y soluciones que apoyen al desarrollo equilibrado del territorio.

\section{RESULTADOS}

Después del terremoto del 16 de abril del 2016, el $80 \%$ de las viviendas de la parroquia se destruyeron o quedaron inhabilitadas; el $20 \%$ estaban afectadas, pero había la posibilidad de recuperarlas o repararlas. Con el movimiento telúrico del 18 de mayo del 2016, la

\footnotetext{
2 Tomado de Tabla 31.- Proyecciones referenciales de población Período 2010 -
} 2020, PDyOT 2016 
situación empeoró, las casas sentidas y/o cuarteadas, se terminaron de caer, lo cual puso en evidencia varios problemas ligados a: la falta de planificación y control en el uso y ordenamiento del suelo, sistemas constructivos antitécnicos y materiales inadecuados; muchas viviendas estaban asentadas en zonas de riesgo y áreas de poca seguridad como rellenos en los márgenes del estero.

Esta información nos arroja la necesidad de generar productos tangibles, resultados propositivos que mejoren la calidad de vida de forma integral de todos los habitantes. Para determinar la estructuración del proyecto, se realizaron diagnósticos en detalle que generaron los siguientes lineamientos macro:
a) Factor Biofísico
b) Factor Riesgos
c) Factor Sociocultural
d) Factor Económico Productivo
e) Factor Asentamientos Humanos
f) Factor Movilidad, Energía y Conectividad
g) Factor Político institucional y participación ciudadana

Basados en estos lineamientos se crearon 11 componentes propios del proyecto en sí que se detallan:

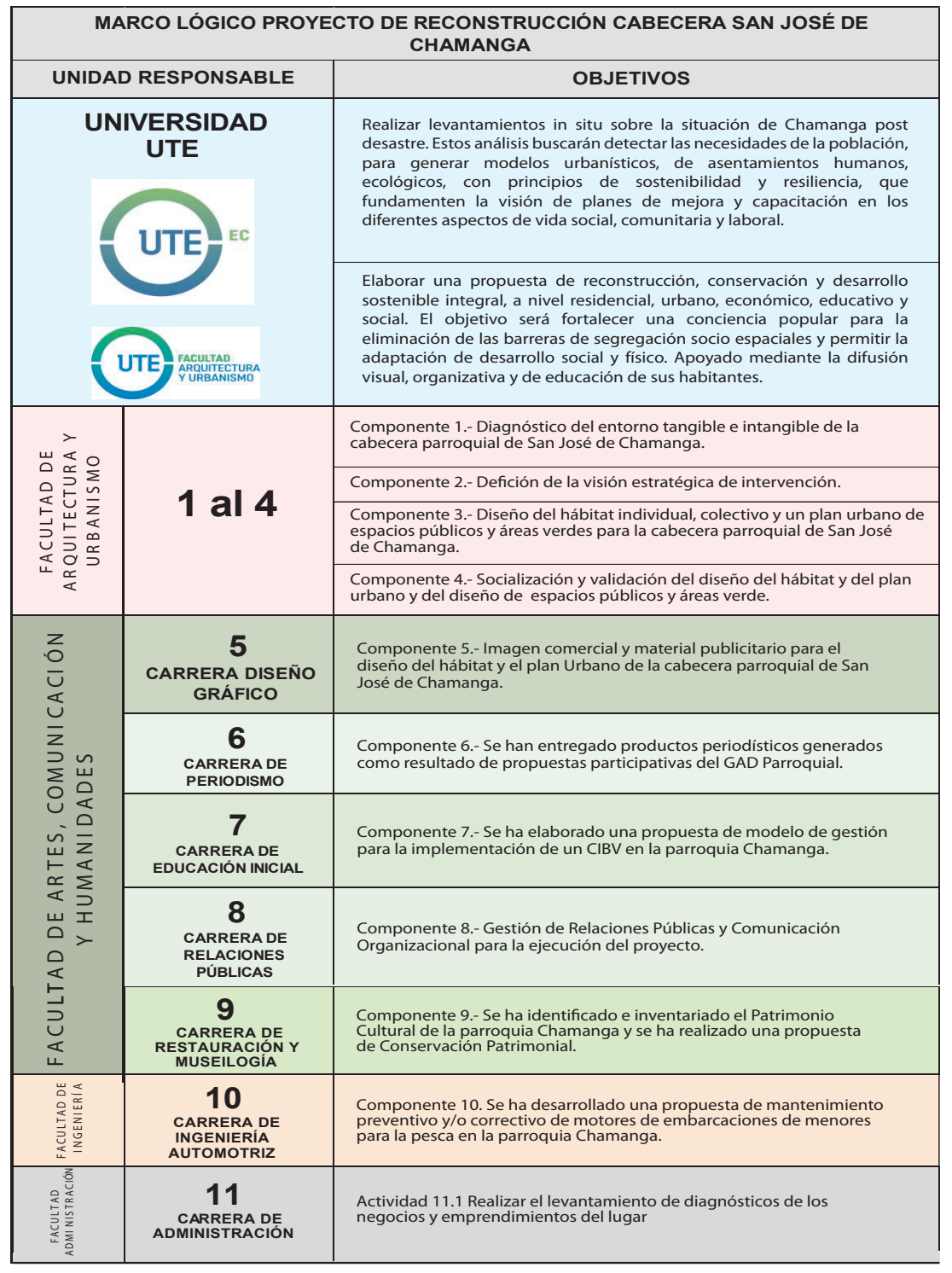

Fuente: Coordinación de Vinculación -

Facultad de Arquitectura y Urbanismo Universidad UTE. 
Sobre este proyecto a la fecha actual se ha alcanzado un avance real del $85 \%$, generando entregas parciales y cierres satisfactorios de los productos de los componentes 1, 2, 5, 6, 7, 8, 9 y 10 .

Este experimento teórico-práctico, ha cruzado fronteras, no ha quedado limitado únicamente al territorio ecuatoriano. Este trabajo ha sido presentado en el extranjero, generando mucha expectativa y admiración. Es un proyecto cuyo punto de partida se basó en la solidaridad. En la actualidad la palabra solidaridad es muy utilizada, se tiene claro que la meta de esta definición es lograr generar un interés por el bien del prójimo. A nivel país existe una cantidad importante de organismos e instituciones que se interesan por fomentar este valor, entre ellos, las universidades.

\section{CONCLUSIONES Y RECOMENDACIONES}

La desigualdad social, tiene relación directa con la desigualdad económica, por lo tanto se podría decir que este fenómeno es un problema socio-económico, que de acuerdo a los eruditos en estas materias se genera fundamentalmente por una mala distribución de la renta de los países. En otras palabras, desigualdad es la discriminación que afecta de manera directa a un grupo de personas, mientras que al mismo tiempo favorece a otras. Este fenómeno a nivel social, crea desigualdad de género, desigualdad racial, desigualdad regional, entre otros.
Pro lado, la desigualdad aumenta cuando un determinado g a servicios públicos básicos como salud o educación. Todos estos abusos desencadenan en discriminación entre individuos, dificultando su acceso a la educación, tecnología, conocimientos. Incentivando así sentimientos de vulnerabilidad que por consiguiente evolucionarán en actitudes de rechazo, hambre, desnutrición y mortalidad prematura en las poblaciones. Es por esto que la vinculación con la sociedad debe ser tomada en cuenta ya que de todas las actividades dentro del bagaje de la vida universitaria, estos proyectos son los que mayor punto de reflexión y aplicación encontrarán los estudiantes entre las necesidades sociales y académicas. Estos proyectos, debido a su magnitud y demanda de recursos, simplemente no podrían ejecutarse sin el accionar de los estudiantes. Los alumnos empiezan a generar sus propias vivencias y con esto sus criterios formados en base a experiencias tangibles.

Es en los institutos de educación donde recae la responsabilidad para crear proyectos solidarios y fomentar la participación por parte de los estudiantes, futuros profesionales del país. En la gran mayoría de casos se confunde voluntariado con vinculación. Si bien es cierto tienen un lineamiento parecido, el alcance de cada trabajo es lo que diferencia estos términos. Sin embargo, es importante comprender que el factor que tienen en común todos estos proyectos es únicamente la solidaridad. Este valor es la esencia del ser humano por excelencia. Esto debe ser el punto de partida para cualquier objetivo de los futuros profesionales que se están formando en las Universidades.

De acuerdo a la experiencia adquirida en este proyecto se podría determinar que la solidaridad tiene fundamentos humanos, tales como igualdad, necesidad de apoyo, fraternidad, dignidad común. La solidaridad ha demostrado no tener banderas, límites territoriales, culturales, religiosos, tendencias políticas. Más que un acto social, es 
una situación que le permite al ser humano mantener y mantenerse en su naturaleza de ser humano.

El enfoque que toma la Universidad UTE en este proyecto puntual de San José de Chamanga, tiene gran importancia, ya que los docentes y estudiantes han descubierto que no todo se reduce a estudiar una carrera. Por lo tanto, se recomienda que la educación en valores, sea un pilar fundamental para que el Ecuador siembre la semilla de un futuro prometedor cimentado en el compromiso social colectivo. Las universidades tienen un fuerte compromiso social. Es por todos estos argumentos que tanto las universidades como los institutos de educación superior, deben ser tomados más en cuenta, la mano de obra calificada con la que cuentan estos centros es de muchísimo valor. Se puede ayudar a cientos o miles de personas, gracias al esfuerzo conjunto de cada uno de sus miembros.

\section{REFERENCIAS BIBLIOGRÁFICAS}

Amaya, L. (2014 de octubre de 2014). De Bogotá a Bombay: prácticas de vivienda informal y desarrollo progresivo. Obtenido de Plataformaarquitectura: http://www. plataformaarquitectura.cl/ cl/755959/de-bogota-a-bombay- practicas-de-vivienda-informal-ydesarrollo-progresivo

Grunaguer, S. (2010). The person as an individual element. Journal LC.
Montoya, N. (2014). EL URBANISMO SOCIAL: LAS TÁCTICAS DE UN ESTADO ANFIBIO. Medellín.

Moya, R. (Febrero de 2019). Reconstrucción de la cabecera parroquial de San José de Chamanga, Arquitectura en Movimiento. (F. León, Entrevistador)

Murray, S. (1992). TEORÍA ELEMENTAL DEL MUESTREO, TEORÍA DE LA DECISIÓN.

Porreca, R. (Febrero de 2019). Reconstrucción de la cabecera parroquial de San José de Chamanga, Arquitectura en Movimiento. (F. León, Entrevistador)

Ricardo Jordán, D. S. (2011). Guía de la Gestión Urbana. United Nations Publications.

Rocchio, D. (Febrero de 2019). Reconstrucción de la cabecera parroquial de San José de Chamanga, Arquitectura en Movimiento. (F. León, Entrevistador)

Rojo, S. (201 1). Herramientas para evaluar la sostenibilidad. Informes de la Construcción, Edicion 41 a 49.

Tellería, I. (2012). Los movimientos urbanos como impulsadores de la gestión democrática en la ciudad. Pamplona, España: Universidad del país Vasco. 